\title{
Cervical Epidural Hematoma after Chiropractic Spinal Manipulation Therapy in a Patient with an Undiagnosed Cervical Spinal Arteriovenous Malformation
}

Meng Huang ${ }^{1}$, Sean M. Barber ${ }^{1}$, Marc Moisi ${ }^{2}$, Suzanne Powell ${ }^{3}$, Andreana Rivera ${ }^{3}$, Michael Zwillman ${ }^{4}$, James Rose ${ }^{5}$

1. Department of Neurosurgery, Houston Methodist Neurological Institute 2. Neurosurgery, Seattle Science Foundation 3. Department of Pathology and Laboratory Medicine, Houston Methodist Hospital 4. Anesthesia and Critical Care, Houston Methodist Willowbrook Hospital 5. Department of Neurosurgery, University Medical Center Brackenridge

$\square$ Corresponding author: Michael Zwillman, zwillman@earthlink.net Disclosures can be found in Additional Information at the end of the article

\section{Abstract}

Spinal epidural hematoma (SEH) occurring after chiropractic spinal manipulation therapy (CSMT) is a rare clinical phenomenon. Our case is unique because the patient had an undiagnosed cervical spinal arteriovenous malformation (AVM) discovered on pathological analysis of the evacuated hematoma. Although the spinal manipulation likely contributed to the rupture of the AVM, there was no radiographic evidence of the use of excessive force, which was seen in another reported case. As such, patients with a known AVM who have not undergone surgical intervention should be cautioned against symptomatic treatment with CSMT, even if performed properly. Regardless of etiology, SEH is a surgical emergency and its favorable neurological recovery correlates inversely with time to surgical evacuation.

Categories: Neurosurgery

Keywords: spinal epidural hematoma, chiropractic manipulation, spinal arteriovenous malformation

\section{Introduction}

Spinal epidural hematoma (SEH) is a rare phenomenon. Although the etiology of spontaneous SEH is poorly understood, a close association with trauma, coagulopathy, and iatrogenic causes, such as spine surgery, epidural catheterization, and lumbar punctures has been observed with non-spontaneous SEH. Even more rare is chiropractic spinal manipulation therapy (CSMT)-induced SEH, and to the best of our knowledge, there are currently only 11 other published cases [1-11]. Our case is unique because the patient had an undiagnosed cervical spinal arteriovenous malformation (AVM) revealed on pathological specimens from the evacuated hematoma.

\section{Case Presentation}

Informed patient consent was obtained from the patient described in this case presentation. No identifying patient information is contained in this paper.

The patient is a 40 -year-old male with no significant medical history who presented with a one-day history of severe neck pain and weakness of the left arm and leg. He underwent CSMT 


\section{Cureus}

the day prior to admission, and the onset of pain occurred immediately following the CSMT session. He was not taking warfarin (Coumadin), aspirin, clopidogrel (Plavix), or any other anticoagulation agents. Admission INR was 1.0 and his platelet count was 320,000. The patient reported a slight decreased sensation weakness on his left side that began acutely approximately five hours prior to coming to the hospital. On physical examination, he was found to have marked weakness of the left upper extremity. His left triceps strength was $4 / 5$, his grip, finger extension, wrist flexion, and extension were all 3/5. The motor strength of the left lower extremity was between 3 to 4 out of 5 throughout. The strength of both right upper and lower extremities was fully intact. An MRI of the spine identified a large posterior epidural hematoma extending from $\mathrm{C} 2$ to $\mathrm{T} 2$, displacing the cord anteriorly and laterally to the right (Figures 1-2).

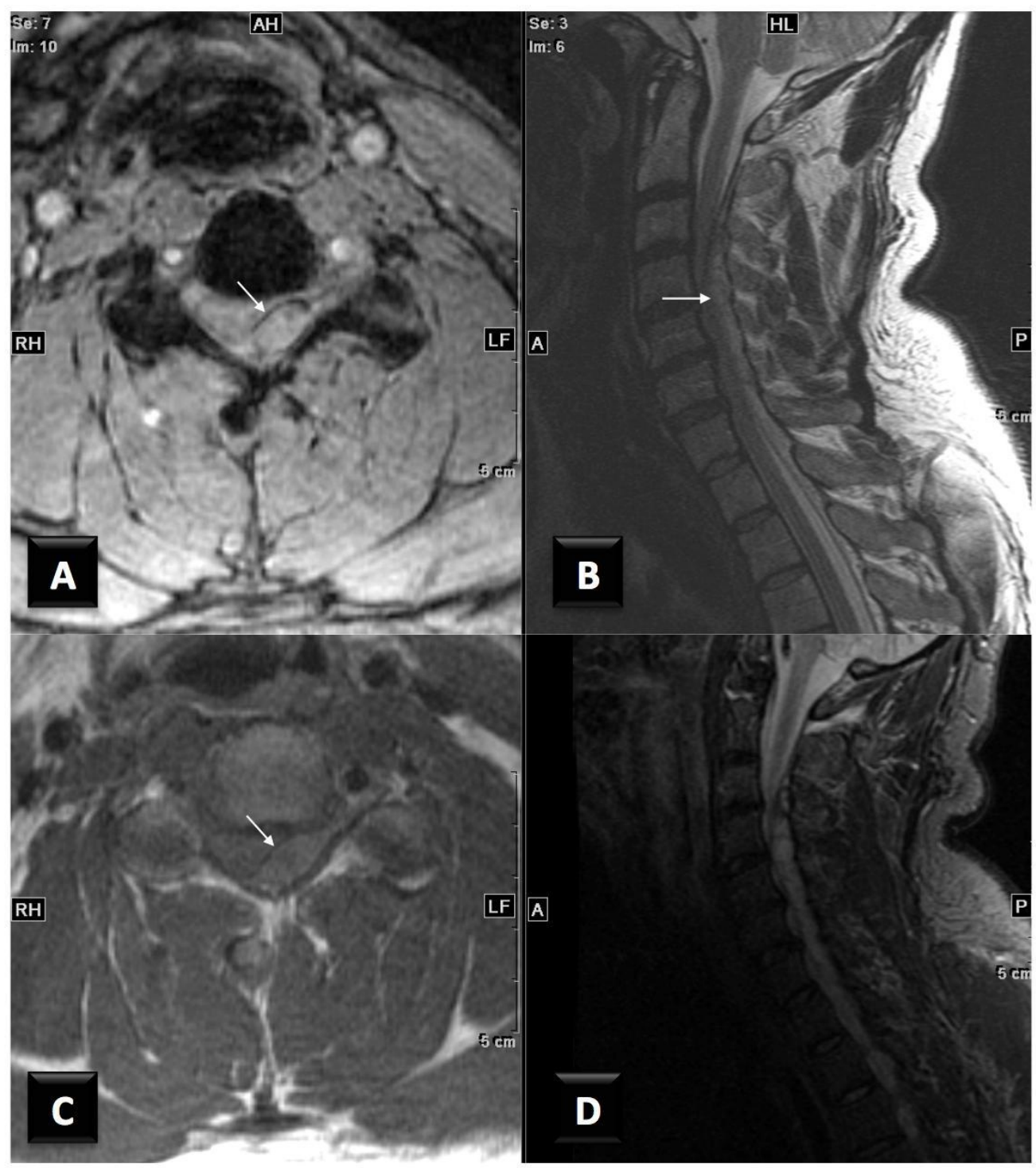

\section{FIGURE 1: A) Axial T2 GRE image, B) Sagittal T2 FSE image, C) Axial T1 SE without contrast.}

The images show an extradural heterogenous collection (arrows) in the left posterior epidural space, which extends from $\mathrm{C} 2$ to $\mathrm{C6}$, compressing the thecal sac and spinal cord. The mass demonstrates heterogenous T2 signal and isointense to slightly increased T1 signal centrally with rim of susceptibility representing blood products in an epidural hematoma most likely in the acute to early subacute stage. 


\section{Cureus}

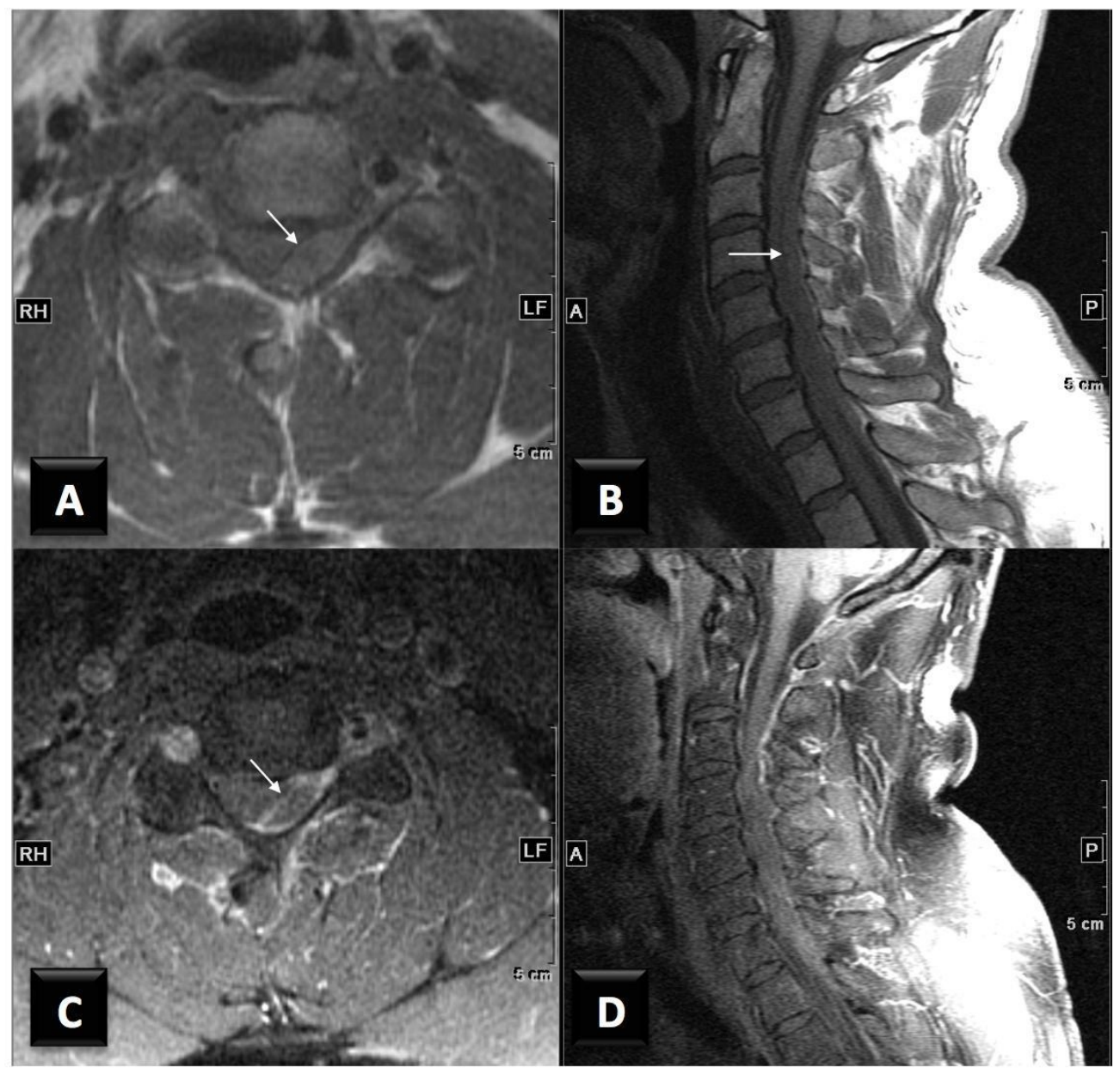

\section{FIGURE 2: A and B) Axial and sagittal T1 SE images without contrast, C and D) Axial and sagittal T1 SE images with contrast and fat saturation.}

The images show the extradural heterogenous collection in the left posterior epidural space, which demonstrates isointense to slightly increased T1 signal with minimal peripheral enhancement (arrows), displacing the spinal cord laterally.

A CT angiogram showed normal cervical vasculature. A formal spinal angiogram was not performed due to the development of a left hemiplegia necessitating surgical intervention. Approximately 18 hours after initial radiologic imaging, a left C3-T1 hemilaminectomy was performed with a solid hematoma evacuation. There was no obvious source of active bleeding found at the time of surgery. A specimen from the hematoma was sent for pathological evaluation. Pathologic examination of the hematoma specimen highlighted an arterial component utilizing Verhoeff-Von Giesen (VVG) staining for elastic fibers. The patient was found to have a spinal AVM (Figure $3 a-3 b$ ). Postoperatively, his strength improved to $4 / 5$ in his left upper extremity and $4+/ 5$ is his left lower extremity. He was able to ambulate on his own by postoperative day 3 and was discharged the following day. A follow-up digital subtraction spinal angiogram two months postoperatively failed to identify any other vascular abnormalities. The patient had a minor cerebral thromboembolic event after the angiography, which completely resolved shortly afterwards with medical management. 


\section{Cureus}
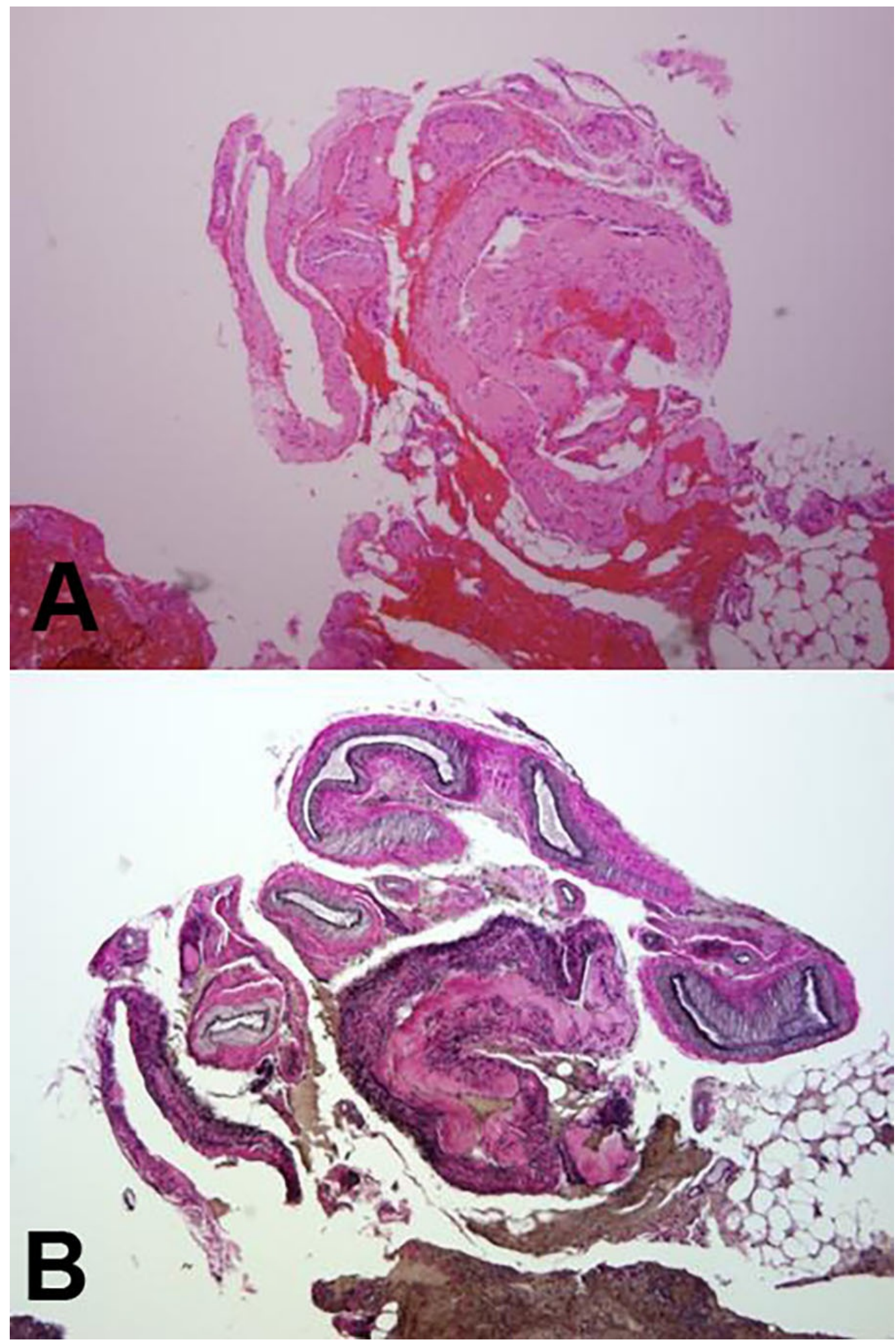

FIGURE 3: Pathologic specimens and staining pattern

A) Hematoxylin and eosin stains of AVF displays a collection of variable caliber blood vessels, some with identifiable internal elastic laminae. B) Verhoeff - van Gieson (VVG) of AVM highlights the internal elastic laminae 


\section{Discussion}

Spinal epidural hematoma results from the rupture of delicate epidural venous plexus veins that are contained between the dural sac and the periosteum of the spinal canal. Because the dural sac and periosteum are adherent in the ventral aspect of the canal, most epidural bleeds occur in the dorsal aspect where the epidural space is relatively slack with loose fatty tissue [12]. Spinal epidural hematomas cause acute compression of the spinal cord, which can present with pain, motor weakness, sensory loss, and bowel and/or bladder dysfunction. The symptoms usually develop within hours to days of the inciting event [13]. MR imaging is the gold standard for diagnosis, evaluation of extension, and assessment of the severity of cord compression [14].

Chiropractic spinal manipulation involves high velocity thrusting forces applied to the spinal column [15-16]. It is hypothesized that these sudden low amplitude thrusts can generate spikes of intraspinal pressure sufficient to cause the rupture of the delicate dural veins [17]. Due to the close temporal association of CSMT and acute onset of severe neck pain and neurologic symptoms, it is likely that our patient's epidural hemorrhage was a result of the chiropractic treatment he underwent the day prior to his presentation. Domenicucci, et al. demonstrated increased signal intensity in T1 and T2 weighted images as well as gadolinium enhancement of the paravertebral muscle mass consistent with a hemorrhagic contusion. They concluded that this finding was suggestive of excessive force attributable to improper CSMT [1]. In our case, the patient's MR imaging did not demonstrate evidence of excessive force. This suggests that even if performed properly, standard CSMT can generate enough intraspinal force to precipitate rupture of a spinal AVM that is already under pressure within the canal. We, therefore, caution that treatment with CSMT should be avoided in patients who have a known spinal AVM causing neck pain and myeloradiculopathy prior to surgical/endovascular obliteration.

Although the spontaneous resolution of a cervical SEH has been reported in the literature [1819], the consensus is that symptomatic SEH is a surgical emergency and early operation is the key to neurological recovery. McQuarrie observed that surgical treatment provided within the first 36 hours of symptom onset results in a favorable prognosis of adequate recovery. Beyond that time period, chances of a good outcome fall drastically, and if surgery is delayed over 3.5 days, patients fail to recover any voluntary movement by five months [20]. Based on the experience of two cases, Song and Lee reported poor outcomes if surgery was performed after 24 hours from symptom onset [9].

Lawton, et al. investigated a series of 30 patients who suffered from a spinal epidural hematoma and examined the relationship between surgical timing and neurological outcome. The average postoperative Frankel grade decreased from 4.7 to 3.7 in patients operated on in less than six hours versus after 24 hours, and there was a corresponding decrease in rate of complete recovery from $67 \%$ to $12 \%$. Better outcomes were seen in patients operated on within 12 hours of symptom onset [21].

\section{Conclusions}

Spinal epidural hematoma is a rare surgical emergency that is associated with trauma, spondylosis, coagulopathy, and iatrogenic procedures, such as spine surgery and lumbar punctures. Presentation following manipulation of the spine is even more rare. To the best of our knowledge, SEH following CSMT in a patient with an undiagnosed cervical spinal AVM has never been reported. It is possible that even properly performed, non-excessive CSMT may precipitate spinal AVM rupture; therefore, patients with known spinal AVM should avoid CSMT for symptom relief prior to surgical/endovascular intervention. Regardless of etiology, SEH causes severe compression of the cord, which can result in irreversible neurological deficits. Because the likelihood of complete neurological recovery is inversely correlated with the time 
to surgical intervention, a high index of suspicion, early recognition, and evacuation is essential.

\section{Additional Information \\ Disclosures}

Human subjects: Consent was obtained by all participants in this study. Conflicts of interest: In compliance with the ICMJE uniform disclosure form, all authors declare the following:

Payment/services info: All authors have declared that no financial support was received from any organization for the submitted work. Financial relationships: All authors have declared that they have no financial relationships at present or within the previous three years with any organizations that might have an interest in the submitted work. Other relationships: All authors have declared that there are no other relationships or activities that could appear to have influenced the submitted work.

\section{References}

1. Domenicucci M, Ramieri A, Salvati M, Brogna C, Raco A: Cervicothoracic epidural hematoma after chiropractic spinal manipulation therapy. Case report and review of the literature. J Neurosurg Spine. 2007, 7:571-74. 10.3171/SPI-07/11/571

2. Garza-Mercado R: Traumatic extradural hematoma of the cervical spine. Neurosurg. 1989, 24:410-14. 10.1227/00006123-198903000-00018

3. Heiner JD: Cervical epidural hematoma after chiropractic spinal manipulation . Am J Emerg Med. 2009, 27:1023.e1-2. 10.1016/j.ajem.2008.12.031

4. Lidder S, Lang KJ, Masterson S, Blagg S: Acute spinal epidural haematoma causing cord compression after chiropractic neck manipulation: an under-recognised serious hazard?. J R Army Med Corps. 2010, 156:255-57. 10.1136/jramc-156-04-11

5. Niedhart CH: Chiropractic manipulation and spinal epidural hematoma--what came first? . Z Orthop Ihre Grenzgeb. 2004, 142:631-32. 10.1055/s-2004-832324

6. Saxler G, Barden B: Extensive spinal epidural hematoma--an uncommon entity following cervical chiropractic manipulation. Z Orthop Ihre Grenzgeb. 2004, 142:79-82. 10.1055/s-2004818032

7. Segal DH, Lidov MW, Camins MB: Cervical epidural hematoma after chiropractic manipulation in a healthy young woman: case report. Neurosurg. 1996, 39:1043-45. 10.1097/00006123-199611000-00034

8. Solheim O, Jorgensen JV, Nygaard OP: Lumbar epidural hematoma after chiropractic manipulation for lower-back pain: case report. Neurosurg. 2007, 61:E170-1. 10.1227/01.neu.0000279740.61048.e2

9. Song KJ, Lee KB: The poor outcome of the delayed diagnosis of acute spontaneous spinal epidural hematoma: two cases report. J Korean Med Sci. 2005, 20:331-34. 10.3346/jkms.2005.20.2.331

10. Tseng SH, Chen Y, Lin SM, Wang CH: Cervical epidural hematoma after spinal manipulation therapy: case report. J Trauma. 2002, 52:585-86. 10.1097/00005373-200203000-00033

11. Whedon JM, Quebada PB, Roberts DW, Radwan TA: Spinal epidural hematoma after spinal manipulative therapy in a patient undergoing anticoagulant therapy: a case report. J Manipulative Physiol Ther. 2006, 29:582-85. 10.1016/j.jmpt.2006.06.017

12. Patel H, Boaz JC, Phillips JP, Garg BP: Spontaneous spinal epidural hematoma in children . Pediatr Neurol. 1998, 19:302-7. 10.1016/S0887-8994(98)00059-9

13. Cakir E, Karaarslan G, Usul H, Baykal S, Kuzeyli K, Mungan I, Yazar U, Peksoylu B, Aynaci M, Cakir F: Clinical course of spontaneous spinal epidural haematoma mimicking Guillain-Barre syndrome in a child: a case report and literature review. Dev Med Child Neurol. 2004, 46:83842. 10.1017/S001216220400146X

14. Sklar EM, Post JM, Falcone S: MRI of acute spinal epidural hematomas. J Comput Assist Tomogr. 1999, 23:238-43. 10.1097/00004728-199903000-00012

15. DiFabio RP: Manipulation of the cervical spine: Risks and benefits. Phys Ther. 1999, 79:5065. 


\section{Cureus}

16. Ernst E: Adverse effects of spinal manipulation: a systematic review . J R Soc Med. 2007, 100:330-38. 10.1258/jrsm.100.7.330

17. Pan G, Kulkarni M, MacDougall DJ, Miner ME: Traumatic epidural hematoma of the cervical spine: diagnosis with magnetic resonance imaging. J Neurosurg. 1988, 68:798-801.

18. Rechtine GR 2nd, Bolesta MJ, Chrin AM, Louis K: Spontaneous resolution of symptomatic post-traumatic cervical epidural hematoma. A case report. J Bone Joint Surg Am. 2001, 83A:255-58.

19. Sei A, Nakamura T, Hashimoto N, Mizuta H, Sasaki A, Takagi K: Cervical spinal epidural hematoma with spontaneous remission. J Spinal Disord. 1991, 4:234-37.

20. McQuarrie IG: Recovery from paraplegia caused by spontaneous spinal epidural hematoma . Neurology. 1978, 28:224-28. 10.1212/WNL.28.3.224

21. Lawton MT, Porter RW, Heiserman JE, Jacobowitz R, Sonntag VK, Dickman CA: Surgical management of spinal epidural hematoma: relationship between surgical timing and neurological outcome. J Neurosurg. 1995, 83:1-7. 10.3171/jns.1995.83.1.0001 